\title{
Linear-Parameter-Varying Antiwindup Compensation for Enhanced Flight Control Performance
}

\author{
Bei $\mathrm{Lu}^{*}$ and $\mathrm{Fen} \mathrm{Wu}^{\dagger}$ \\ North Carolina State University, Raleigh, North Carolina 27695 \\ and \\ SungWan Kim ${ }^{\ddagger}$ \\ NASA Langley Research Center, Hampton, Virginia 23681
}

\begin{abstract}
Actuator saturation is one of the major issues of flight control in the high angleof-attack region. This paper presents a saturation control scheme for linear parametervarying (LPV) systems from an antiwindup control perspective. The proposed control approach is advantageous from the implementation standpoint because it can be thought of as an augmented control algorithm to the existing control system. Moreover, the synthesis condition for an antiwindup compensator is formulated as a linear matrix inequality (LMI) optimization problem and can be solved efficiently. We have applied the LPV antiwindup controller to an F-16 longitudinal autopilot control system design and compared it with the thrust vectoring control scheme. The nonlinear simulations show that an LPV antiwindup controller improves flight quality and offers advantages over thrust vectoring in a high angle-of-attack region.
\end{abstract}

\section{Introduction}

$\mathbf{T}$ HE flight control system of a tactical aircraft has different performance goals for low angle-ofattack and high angle-of-attack regions. For example, pilots desire fast and accurate responses for maneuver and attitude tracking in a low angle-of-attack scenario. While in a high angle-of-attack region, the flight control focuses on the maintainability of aircraft stability with acceptable flying qualities. The potential of high angle-of-attack flight presents many challenges to the control designers. Because of aerodynamic surface saturation and control surface limitation, unconventional actuators such as thrust vectoring are suggested for aircraft maneuvering at and beyond the stall angle-ofattack. However, incorporation of additional thrust vectoring hardware could complicate the design of flight control laws in the poststall regime. ${ }^{1}$ Robust multivariable control methods have been recently applied to a variety of aircraft models ${ }^{2}$ to demonstrate their abilities to fly at high angles-of-attack with the help of thrust vectoring control (see Ref. 3 and references therein). Besides control law design, another major issue of high angle-of-attack flight is control saturation. It is well recognized that actuator saturation degrades the performance of the flight control system and can even lead to instability. The destabi-

*Ph.D Student, Dept. of Mechanical and Aerospace Engineering. Student Member AIAA.

$\dagger$ Assistant Professor, Dept. of Mechanical and Aerospace Engineering; fwu@eos.ncsu.edu. Member AIAA.

$\stackrel{\ddagger}{\ddagger}$ Research Engineer, Dynamics and Control Branch. Associate Fellow AIAA. lizing effects of actuator saturation have been cited as contributing factors in several mishaps involving high-performance aircraft. ${ }^{4}$ For this reason, various methods of preventing instability because of saturation have been examined, which include allocating control effectors and command scaling and prioritization.

Antiwindup control method is a popular approach to control saturation that employs a two-step design procedure. The main idea of antiwindup control is to design the linear controller by ignoring the saturation nonlinearities first and then add antiwindup compensation to minimize adverse effects of the saturation on closed-loop performance. Desirable design requirements for antiwindup compensation subject to actuator saturation are the closed-loop system stability, recovery of the linear design specifications in the absence of saturation (linear performance recovery), and the smooth degradation of the linear performance in the presence of saturation (graceful performance degradation). Like other saturation control techniques, the antiwindup compensator design often assumes a linear time-invariant (LTI) plant and models the saturation block as a sector-bounded nonlinearity. Then absolute stability conditions (such as Popov, circle theorems) are applied for the stability and performance analysis. ${ }^{5}$ Their extension to nonlinear systems has already been developed.

A general framework that unifies a large class of existing antiwindup control schemes in terms of two matrix parameters was proposed in Ref. 6. This framework is useful for understanding different antiwindup control schemes and motivates the devel- 
opment of systematic procedures for designing antiwindup controllers that provide guaranteed stability and performance. Early results in antiwindup control often have the drawback of lacking rigorous stability analysis and clear exposition of performance objectives. Using an extended circle criterion, the synthesis condition of static antiwindup controllers is formulated as a linear matrix inequality (LMI) problem. $^{7}$ A recent study in Ref. 8 has further revealed that antiwindup control for stable open-loop LTI systems can be solved globally as an LMI problem with the order of antiwindup compensator no more than the plant's order. Alternatively, the Popov stability condition has also been applied to the antiwindup compensator design problem. ${ }^{9}$ However, the synthesis condition of the saturation controller is given in coupled Riccati equations, which are difficult to solve for the optimal solution. Most previous antiwindup compensator designs are only applicable to open-loop stable LTI systems, limiting their usefulness for practical problems. When the system is nonlinear and open-loop unstable, the control synthesis problem becomes very difficult to solve; therefore, global stabilization cannot be achieved. ${ }^{10,11}$ However, in many control systems including flight control systems, the system dynamics are inherently nonlinear and their linearizations at some operation points are strictly unstable.

The motivation for this research is twofold. First, the antiwindup control scheme for LTI plants in Ref. 12 is generalized to linear parameter-varying (LPV) systems. This generalization is very important because of the relevance of LPV systems to nonlinear systems. In fact, the LPV model can be thought of as a group of local descriptions of nonlinear dynamics. The antiwindup compensation augments existing control systems by maintaining stability and recovering control performance when actuators become saturated. Second, saturation control for aircraft under large maneuver operations is critical because of safety concerns. The proposed antiwindup compensation can augment existing flight control algorithms, yielding enhanced reliability and an expanded flight envelope. In particular, using an F-16 longitudinal dynamic model, we demonstrate good flight control performance of LPV antiwindup control in a high angle-of-attack scenario. Note that the study in this paper is focused on actuator magnitude saturations, and it is the first, but an important step towards the actual application of antiwindup to high-performance flight control. The results can be easily generalized to actuator rate saturations, ${ }^{13}$ which are more realistic for an advanced tactical aircraft.

The notation in this paper is standard. $\mathbf{R}$ stands for the set of real numbers and $\mathbf{R}_{+}$for the non-negative real numbers. $\quad \mathbf{R}^{m \times n}$ is the set of real $m \times n$ matrices. The transpose of a real matrix $M$ is denoted by $M^{T} . \operatorname{Ker}(M)$ is used to denote the orthogonal complement of $M$. A block diagonal matrix with submatrices $X_{1}, X_{2}, \ldots, X_{p}$ in its diagonal is denoted by $\operatorname{diag}\left\{X_{1}, X_{2}, \ldots, X_{p}\right\}$. We use $\mathbf{S}^{n \times n}$ to denote the real symmetric $n \times n$ matrices and $\mathbf{S}_{+}^{n \times n}$ to denote positive definite matrices. If $M \in \mathbf{S}^{n \times n}$, then $M>0(M \geq 0)$ indicates that $M$ is positive definite (positive semidefinite) and $M<0(M \leq 0)$ denotes a negative definite (negative semidefinite) matrix. If $a, b \in \mathbf{R}$, then $\operatorname{sect}[a, b]$ denotes the conic sector defined by $\{(q, p):(p-a q)(p-b q) \leq 0\}$. For $x \in \mathbf{R}^{n}$, its norm is defined as $\|x\|:=\left(x^{T} x\right)^{\frac{1}{2}}$. The space of square integrable functions is denoted by $\mathcal{L}_{2}$, that is, for any $u \in \mathcal{L}_{2}$,

$$
\|u\|_{2}:=\left[\int_{0}^{\infty} u^{T}(t) u(t) d t\right]^{\frac{1}{2}}
$$

is finite.

This paper is organized as follows: Section II is devoted to providing an LPV antiwindup compensator synthesis condition, which is a generalization of previous results in Ref. 12 . In section III, the LPV antiwindup compensator is applied to an F-16 longitudinal flight control system design and is compared with the thrust vectoring control scheme. Finally, we conclude the paper in section IV.

\section{LPV Antiwindup Control Synthesis}

The goal of antiwindup compensation is to modify nominal controllers so that if the signal from the controller is different than that which enters the plant, corrective feedback action is employed to reduce the discrepancy. Because it is impossible to provide a global stabilizing solution to the antiwindup control problem when the open-loop plant is unstable, one often needs to determine regional stability for saturation control and to design the controller gains in the guaranteed stability region. ${ }^{10,11}$ In Ref. 8, a sectorbounded input nonlinearity, sect $[0,1]$, was considered for the open-loop stable plant and is not applicable to exponentially unstable systems. However, the derived performance and stability properties can be improved when the input nonlinearity is restricted to a smaller sector region. As a result, this modification leads to regional stability of the antiwindup compensated system and extends the antiwindup control technique to exponentially unstable open-loop systems.

Consider an LPV plant $P_{\rho}$ described by

$$
\left[\begin{array}{c}
\dot{x}_{p} \\
e \\
y
\end{array}\right]=\left[\begin{array}{ccc}
A_{p}(\rho) & B_{p 1}(\rho) & B_{p 2}(\rho) \\
C_{p 1}(\rho) & D_{p 11}(\rho) & D_{p 12}(\rho) \\
C_{p 2}(\rho) & D_{p 21}(\rho) & D_{p 22}(\rho)
\end{array}\right]\left[\begin{array}{c}
x_{p} \\
d \\
\sigma(u)
\end{array}\right]
$$

where the plant state $x_{p} \in \mathbf{R}^{n_{p}} . y \in \mathbf{R}^{n_{y}}$ is the measurement for control, and $\sigma(u) \in \mathbf{R}^{n_{u}}$ is the saturated control input. $e \in \mathbf{R}^{n_{e}}$ is the controlled output and $d \in \mathbf{R}^{n_{d}}$ is the disturbance input. It is assumed 
that the vector-valued parameter $\rho$ evolves continuously over time and its range is limited to a compact subset $\mathcal{P} \subset \mathbf{R}^{s}$. In addition, its time derivative is assumed to be bounded and satisfy the constraint $\underline{\nu}_{i} \leq \dot{\rho}_{i} \leq \bar{\nu}_{i}, i=1,2, \ldots, s$. For notational purposes, denote $\mathcal{V}=\left\{\nu: \underline{\nu}_{i} \leq \nu_{i} \leq \bar{\nu}_{i}, i=1,2, \ldots, s\right\}$, where $\mathcal{V}$ is a given convex polytope in $\mathbf{R}^{s}$ that contains the origin. Given the sets $\mathcal{P}$ and $\mathcal{V}$, the parameter $\nu$ variation set is defined as

$\mathcal{F}_{\mathcal{P}}^{\nu}=\left\{\rho \in \mathcal{C}^{1}\left(\mathbf{R}_{+}, \mathbf{R}^{s}\right): \rho(t) \in \mathcal{P}, \dot{\rho}(t) \in \mathcal{V}, \forall t \geq 0\right\}$

So $\mathcal{F}_{\mathcal{P}}^{\nu}$ specifies the set of all allowable parameter trajectories.

All matrix valued state-space data are continuous and have appropriate dimensions. For simplicity, we assume that

(A1) $\left(A_{p}(\rho), B_{p 2}(\rho), C_{p 2}(\rho)\right)$ triple is parameterdependent stabilizable and detectable for all $\rho$.

(A2) The matrix functions $\left[B_{p 2}^{T}(\rho) \quad D_{p 12}^{T}(\rho)\right]$ and $\left[\begin{array}{ll}C_{p 2}(\rho) & \left.D_{p 21}(\rho)\right]\end{array}\right.$ have full row rank for all $\rho$.

(A3) $D_{p 22}(\rho)=0$.

The actuator nonlinearity under consideration is a piecewise-linear saturation

$$
\sigma\left(u_{i}\right)= \begin{cases}u_{i} & \left|u_{i}\right| \leq u_{i}^{\max } \\ \operatorname{sgn}\left(u_{i}\right) u_{i}^{\max } & \left|u_{i}\right|>u_{i}^{\max }\end{cases}
$$

for $i=1,2, \ldots, n_{u}$. The antiwindup control structure is shown in Fig. 1(a).

Following the standard antiwindup procedure, a nominal LPV controller $K_{\rho}$ is designed first by ignoring the input nonlinearity. Different control design techniques can be employed to achieve this goal. A systematic way to do this is through LPV control theory. ${ }^{14-16}$ Because of assumption (A1), the nominal controller $K_{\rho}$ exists and is capable of stabilizing the open-loop system when no input saturation exists, and its design will determine the nominal performance of the closed-loop LPV system. We assume that such a controller is given by

$$
\left[\begin{array}{c}
\dot{x}_{k} \\
u
\end{array}\right]=\left[\begin{array}{cc}
A_{k}(\rho, \dot{\rho}) & B_{k}(\rho) \\
C_{k}(\rho) & D_{k}(\rho)
\end{array}\right]\left[\begin{array}{c}
x_{k} \\
y
\end{array}\right]+\left[\begin{array}{c}
v_{1} \\
v_{2}
\end{array}\right]
$$

where $x_{k} \in \mathbf{R}^{n_{k}}$. The variables $v_{1}, v_{2}$ are the auxiliary inputs provided by an antiwindup compensator. They are used to condition the nominal controller when the control input is saturated.

Our objective is to design an LPV antiwindup compensator $A W_{\rho}$ such that the adverse effect of input saturations are minimized in terms of induced $\mathcal{L}_{2}$ norm. The antiwindup compensator is in the form of

$$
\left[\begin{array}{c}
\dot{x}_{a w} \\
\hline v_{1} \\
v_{2}
\end{array}\right]=\left[\begin{array}{ll}
A_{a w}(\rho, \dot{\rho}) & B_{a w}(\rho) \\
C_{a w}(\rho, \dot{\rho}) & D_{a w}(\rho)
\end{array}\right]\left[\begin{array}{c}
x_{a w} \\
q
\end{array}\right]
$$

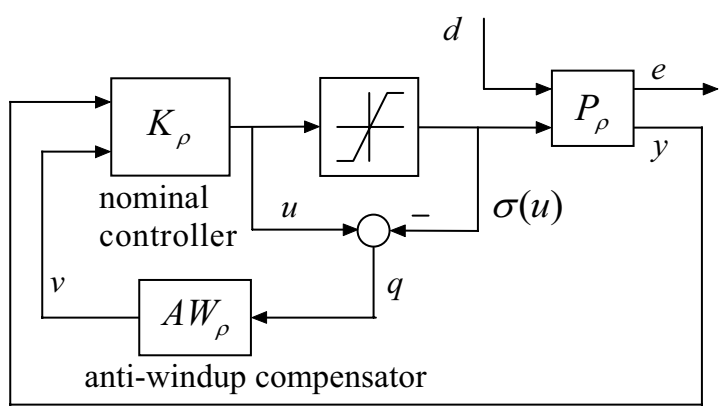

(a) LPV antiwindup control structure

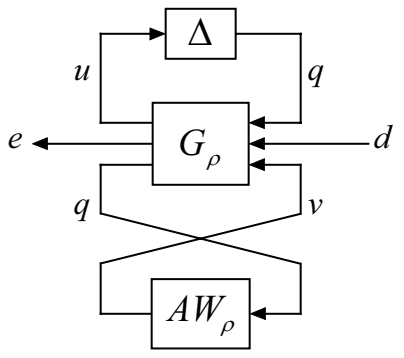

(b) Equivalent transformation

Fig. 1 Nonlinear saturation control diagram.

where the state $x_{a w} \in \mathbf{R}^{n_{a w}}$; the size of the compensator state will be determined in the sequel. Such antiwindup compensation schemes provide a computationally efficient technique for "retro-fitting" existing unconstrained controller $K_{\rho}$ to account for input nonlinearities, thereby eliminating controller windup problems for input saturated nonlinear systems.

The LPV antiwindup control diagram in Fig. 1(a) can be transformed to its equivalent form by substituting each actuator saturation with a deadband nonlinearity $\Delta_{i}=1-\sigma\left(u_{i}\right) / u_{i}$ and $\Delta=$ $\operatorname{diag}\left\{\Delta_{1}, \Delta_{2}, \ldots, \Delta_{n_{u}}\right\}$ as shown in Fig. 1(b). This allows recasting of the compensator design problem into a robust LPV control paradigm.

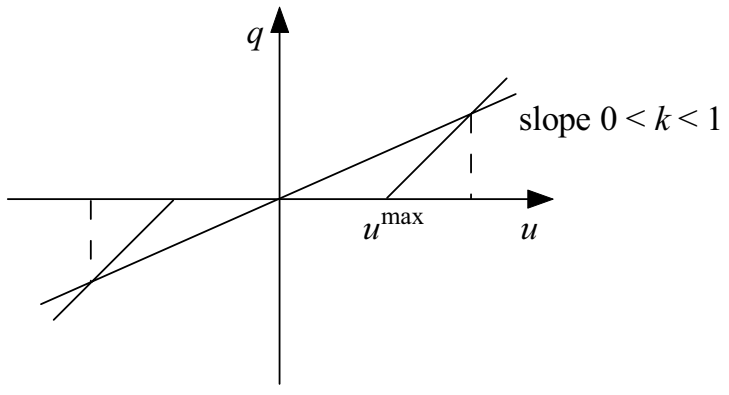

Fig. 2 Restricted range input saturation.

It can be seen that the deadband uncertainty $\Delta_{i}$ resides in the conic sector $[0,1]$. If the maximal value of the control input signal $u_{i}$ is restricted to be less than $\left(1 /\left(1-k_{i}\right)\right) u_{i}^{\max }$ with $0<k_{i}<1$, then the nonlinearity $\Delta_{i}$ is reduced to $\operatorname{sect}\left[0, k_{i}\right]$ as shown in 
Fig. 2. Consequently, we have $\Delta \in \operatorname{sect}[0, K]$ with $K=\operatorname{diag}\left\{k_{1}, k_{2}, \ldots, k_{n_{u}}\right\}$. The resulting stability notion then becomes regional stability, which is more conservative than the actual stability limit. However, this restriction of uncertainty $\Delta$ is capable of extending the antiwindup scheme to open-loop exponentially unstable systems.

Let the system $G_{\rho}$ be the interconnection of the open-loop system $P_{\rho}$ and the nominal controller $K_{\rho}$, but exclude the antiwindup compensator. Then its dynamic equation is

$$
\begin{aligned}
{\left[\begin{array}{c}
\dot{x} \\
u \\
e \\
q
\end{array}\right] } & =\left[\begin{array}{cccc}
A(\rho, \dot{\rho}) & B_{0}(\rho) & B_{1}(\rho) & B_{2}(\rho) \\
C_{0}(\rho) & D_{00}(\rho) & D_{01}(\rho) & D_{02}(\rho) \\
C_{1}(\rho) & D_{10}(\rho) & D_{11}(\rho) & D_{12}(\rho) \\
0 & I & 0 & 0
\end{array}\right]\left[\begin{array}{c}
x \\
q \\
d \\
\hline v_{1} \\
v_{2}
\end{array}\right] \\
q & =\Delta u
\end{aligned}
$$

where $x^{T}=\left[\begin{array}{ll}x_{p}^{T} & x_{k}^{T}\end{array}\right] \in \mathbf{R}^{n}$ with $n=n_{p}+n_{k}$ and $\Delta \in \operatorname{sect}[0, K]$.

$$
\begin{aligned}
A(\rho, \dot{\rho}) & =\left[\begin{array}{cc}
A_{p}+B_{p 2} D_{k} C_{p 2} & B_{p 2} C_{k} \\
B_{k} C_{p 2} & A_{k}
\end{array}\right] \\
B_{0}(\rho) & =\left[\begin{array}{c}
-B_{p 2} \\
0
\end{array}\right], \quad B_{1}(\rho)=\left[\begin{array}{c}
B_{p 1}+B_{p 2} D_{k} D_{p 21} \\
B_{k} D_{p 21}
\end{array}\right] \\
B_{2}(\rho) & =\left[\begin{array}{cc}
0 & B_{p 2} \\
I & 0
\end{array}\right], \quad C_{0}(\rho)=\left[\begin{array}{ll}
D_{k} C_{p 2} & C_{k}
\end{array}\right] \\
C_{1}(\rho) & =\left[\begin{array}{ll}
C_{p 1}+D_{p 12} D_{k} C_{p 2} & D_{p 12} C_{k}
\end{array}\right] \\
D_{00}(\rho) & =0, \quad D_{01}(\rho)=D_{k} D_{p 21} \\
D_{02}(\rho) & =\left[\begin{array}{ll}
0 & I
\end{array}\right], \quad D_{10}(\rho)=-D_{p 12} \\
D_{11}(\rho) & =D_{p 11}+D_{p 12} D_{k} D_{p 21}, \quad D_{12}(\rho)=\left[\begin{array}{ll}
0 & D_{p 12}
\end{array}\right]
\end{aligned}
$$

Note that the state-space data have linear dependency of parameter rate $\dot{\rho}$ under the LPV control design framework.

Denote $x_{c l}^{T}=\left[\begin{array}{ll}x^{T} & x_{a w}^{T}\end{array}\right]$. Then the final closed-loop system $T_{\rho}=\mathcal{F}_{\ell}\left(G_{\rho}, A W_{\rho}\right)$ is described by

$$
\left[\begin{array}{c}
\dot{x}_{c l} \\
u \\
e
\end{array}\right]=\left[\begin{array}{ccc}
A_{c l}(\rho, \dot{\rho}) & B_{0, c l}(\rho) & B_{1, c l}(\rho) \\
C_{0, c l}(\rho, \dot{\rho}) & D_{00, c l}(\rho) & D_{01, c l}(\rho) \\
C_{1, c l}(\rho, \dot{\rho}) & D_{10, c l}(\rho) & D_{11, c l}(\rho)
\end{array}\right]\left[\begin{array}{c}
x_{c l} \\
q \\
d
\end{array}\right]
$$

$$
q=\Delta u
$$

where $\mathcal{F}_{\ell}(\cdot, \cdot)$ stands for a lower linear fractional transformation. ${ }^{17}$ The state-space data of the closed-loop system $T_{\rho}$ are related to the interconnected system $G_{\rho}$ and the antiwindup compensator $A W_{\rho}$. Specifically, the closed-loop state-space data depend on the antiwindup compensator gain in affine form. The following theorem provides a synthesis condition for the antiwindup compensator.
Theorem 1 (synthesis condition for LPV antiwindup compensator)

Given scalars $0<k_{i}<1, i=1,2, \ldots, n_{u}$, the $L P V$ open-loop system $P_{\rho}$ with a parameter-dependent stabilizing nominal controller $K_{\rho}$, if there exists a pair of positive definite matrix functions $R_{11}(\rho) \in \mathbf{S}_{+}^{n_{p} \times n_{p}}$, $S(\rho) \in \mathbf{S}_{+}^{n \times n}$ and a diagonal matrix function $V(\rho)=$ $\operatorname{diag}\left\{v_{1}(\rho), \cdots, v_{n_{u}}(\rho)\right\}>0$ satisfying eqns. (8)-(10) for all $(\rho, \nu) \in \mathcal{P} \times \mathcal{V}$, then there exists an $n_{p}$ th order $L P V$ antiwindup compensator $A W_{\rho}$ to stabilize the closed-loop system exponentially and have the performance $\|e\|_{2}<\gamma\|d\|_{2}$ for all $\rho(\cdot) \in \mathcal{F}_{\mathcal{P}}^{\nu}$ when the condition $\left|u_{i}\right| \leq\left(1 /\left(1-k_{i}\right)\right) u_{i}^{\max }, i=1,2, \ldots, n_{u}$ holds.

The proof can be found in the Appendix. Because only the $(1,1)$ element of the $R$ matrix function is constrained in the LMIs (8)-(10), it is always possible to augment matrix $R_{11}$ to $R$ in satisfying the preceding coupling condition. For example, one can choose

$$
R=\left[\begin{array}{c|l|l}
R_{11}(\rho) & {\left[\begin{array}{ll}
I & 0
\end{array}\right] S^{-1}(\rho)} & {\left[\begin{array}{l}
0 \\
I
\end{array}\right]} \\
\hline\left[\begin{array}{ll}
0 & I
\end{array}\right] S^{-1}(\rho)\left[\begin{array}{l}
I \\
0
\end{array}\right] & {\left[\begin{array}{ll}
0 & I
\end{array}\right] S^{-1}(\rho)\left[\begin{array}{l}
0 \\
I
\end{array}\right]}
\end{array}\right]
$$

The resulting $R$ is positive definite because of condition (10). Also, $R(\rho)-S^{-1}(\rho) \geq 0$ is satisfied for the selected $R$ matrix function. The rank condition is trivially satisfied if one chooses $n_{a w}=n_{p}$.

Recall that the LPV antiwindup compensator synthesis problem is originally formulated as a robust control problem. This usually leads to a nonconvex solvability condition as bilinear matrix inequalities. However, because of the special structure of the antiwindup controller, the resulting synthesis condition is convex in terms of matrix variables $R_{11}(\rho), S(\rho)$, and $V(\rho)$. In fact, the solvability condition for the LPV antiwindup compensator is given as an infinitedimensional LMI optimization problem, for which an efficient numerical algorithm exists to solve it approximately. ${ }^{18}$ This can be done by parameterizing the matrix variables using a finite set of scalar basis functions as

$$
\begin{aligned}
R_{11}(\rho) & =\sum_{i=1}^{N_{f}} f_{i}(\rho) R_{11, i}, \quad S(\rho)=\sum_{j=1}^{N_{g}} g_{j}(\rho) S_{j} \\
V(\rho) & =\sum_{k=1}^{N_{h}} h_{k}(\rho) V_{k}
\end{aligned}
$$

where $\left\{f_{i}(\rho)\right\}_{i=1}^{N_{f}}, \quad\left\{g_{j}(\rho)\right\}_{j=1}^{N_{g}}$, and $\left\{h_{i}(\rho)\right\}_{k=1}^{N_{h}}$ are user-specified scalar basis functions. $R_{11, i}, S_{j}$, and $V_{k}$ are new optimization variables to be determined. After such a parameterization, the LPV synthesis conditions can be solved using a gridding method over the parameter space. 


$$
\begin{aligned}
& {\left[\begin{array}{ccc}
\left\{\begin{array}{c}
R_{11}(\rho) A_{p}^{T}(\rho)+A_{p}(\rho) R_{11}(\rho)-\sum_{i=1}^{s} \nu_{i} \frac{\partial R_{11}}{\partial \rho_{i}} \\
+2 B_{p 2}(\rho) K^{-1} V(\rho)\left(I-K^{-1}\right) B_{p 2}^{T}(\rho)
\end{array}\right\} & \left\{\begin{array}{cc}
R_{11}(\rho) C_{p 1}^{T}(\rho) \\
\left.+2 B_{p 2}(\rho) K^{-1} V(\rho)\left(I-K^{-1}\right) D_{p 12}^{T}(\rho)\right\}
\end{array}\right. & B_{p 1}(\rho) \\
\left\{\begin{array}{c}
C_{p 1}(\rho) R_{11}(\rho) \\
+2 D_{p 12}(\rho) K^{-1} V(\rho)\left(I-K^{-1}\right) B_{p 2}^{T}(\rho)
\end{array}\right\} & \left\{\begin{array}{cc}
-\gamma I_{n_{e}} \\
\left.+2 D_{p 12}(\rho) K^{-1} V(\rho)\left(I-K^{-1}\right) D_{p 12}^{T}(\rho)\right\}
\end{array}\right. & D_{p 11}(\rho) \\
B_{p 1}^{T}(\rho) & D_{p 11}^{T}(\rho) & -\gamma I_{n_{d}}
\end{array}\right]<0} \\
& {\left[\begin{array}{ccc}
S(\rho) A(\rho, \nu)+A^{T}(\rho, \nu) S(\rho)+\sum_{i=1}^{s} \nu_{i} \frac{\partial S}{\partial \rho_{i}} & S(\rho) B_{1}(\rho) & C_{1}^{T}(\rho) \\
B_{1}^{T}(\rho) S(\rho) & -\gamma I_{n_{d}} & D_{11}^{T}(\rho) \\
C_{1}(\rho) & D_{11}(\rho) & -\gamma I_{n_{e}}
\end{array}\right]<0}
\end{aligned}
$$

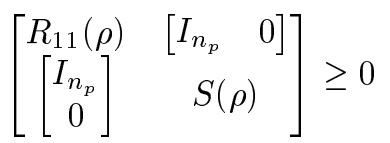

After solving $R_{11}(\rho), S(\rho)$, and $V(\rho)$ matrix functions, the LPV antiwindup compensator gain can be determined by either solving an LMI feasibility problem or the antiwindup compensator can be constructed explicitly as shown in the following theorem. The explicit construction approach is advantageous because it avoids possible numerical ill-conditioning when solving the preceding feasibility LMI problem. Moreover, it connects the antiwindup controller directly to the plant and nominal controller gains.

Theorem 2 (LPV antiwindup compensator construction)

Given the solutions $R_{11}(\rho), S(\rho), \gamma$, and $V(\rho)=$ $W^{-1}(\rho)$ of the LMIs (8)-(10), let $M(\rho) N^{T}(\rho)=I_{n}-$ $R(\rho) S(\rho)$ with $M, N \in \mathbf{S}^{n \times n_{p}}$, and $H^{T}=\left[\begin{array}{ll}I_{n_{p}} & 0\end{array}\right]$, then one $n_{p}$-th order LPV antiwindup compensator $A W_{\rho}$ can be constructed through the following scheme:

1. Compute a feasible $\hat{D}_{a w}(\rho) \in \mathbf{R}^{n_{u} \times n_{u}}$ such that eqn. (11) holds for all $\rho \in \mathcal{P}$,

2. Compute the least square solutions of the linear equations (12)-(13) for $\hat{B}_{a w}(\rho) \in$ $\mathbf{R}^{n \times n_{u}}, \hat{C}_{a w}(\rho) \in \mathbf{R}^{n_{u} \times n_{p}}$, and the matrix $\hat{A}_{a w}(\rho, \dot{\rho}) \in \mathbf{R}^{n \times n_{p}}$ is expressed as in eqn. (14),

3. Convert the transformed antiwindup compensator gain to its original state-space data by eqn. (15).

This proof can be found in the Appendix. The explicit antiwindup construction scheme can also be applied to open-loop stable systems. However, because the synthesis condition for an open-loop stable plant does not involve $W$ (Ref. 8), we need to solve both the feasible $\hat{D}_{a w}$ and $W$ matrix functions at the first step. The remaining steps are the same by setting $K=I$.

\section{Saturation Control for Flight Dynamics}

In this section, the proposed LPV antiwindup control synthesis technique is applied to flight dynamics.
The system to be controlled is the longitudinal F-16 aircraft model based on NASA Langley Research Center (LaRC) wind tunnel tests, ${ }^{19}$ which is described by Stevens and Lewis in great detail. ${ }^{20}$

\section{A. Aircraft Model}

The full nonlinear longitudinal model of an F-16 aircraft is given as follows:

$$
\begin{aligned}
& \dot{V}=\frac{\bar{q} S \bar{c}}{2 m V}\left[C_{x q}(\alpha) \cos \alpha+C_{z q}(\alpha) \sin \alpha\right] q \\
& +\frac{\bar{q} S}{m}\left[C_{x}\left(\alpha, \delta_{e}\right) \cos \alpha+C_{z}\left(\alpha, \delta_{e}\right) \sin \alpha\right] \\
& -g \sin (\theta-\alpha)+T \frac{\cos \alpha}{m} \\
& \dot{\alpha}=\left\{1+\frac{\bar{q} S \bar{c}}{2 m V^{2}}\left[C_{z q}(\alpha) \cos \alpha-C_{x q}(\alpha) \sin \alpha\right]\right\} q \\
& +\frac{\bar{q} S}{m V}\left[C_{z}\left(\alpha, \delta_{e}\right) \cos \alpha-C_{x}\left(\alpha, \delta_{e}\right) \sin \alpha\right] \\
& +\frac{g}{V} \cos (\theta-\alpha)-T \frac{\sin \alpha}{m V} \\
& \dot{q}=\frac{\bar{q} S \bar{c}}{2 I_{y} V}\left[\bar{c} C_{m q}(\alpha)+\Delta C_{z q}(\alpha)\right] q \\
& +\frac{\bar{q} S \bar{c}}{I_{y}}\left[C_{m}\left(\alpha, \delta_{e}\right)+\frac{\Delta}{\bar{c}} C_{z}\left(\alpha, \delta_{e}\right)\right] \\
& \dot{\theta}=q
\end{aligned}
$$

The aerodynamic coefficients are provided as look-up tables from LaRC wind tunnel tests on a scale model of F-16 aircraft. The data apply to the speed range up to about Mach 0.6, and cover a very wide range of angle-of-attack $(-20 \mathrm{deg} \leq \alpha \leq 90 \mathrm{deg})$. However, conventional aerodynamic math models for use in aircraft simulation or flight control design have become increasingly deficient in the poststall region. ${ }^{20}$ So, the investigation on the robust control of aircraft in the high angle-of-attack region with uncertain aerodynamic coefficients is one of the challenging research topics. 


$$
\begin{aligned}
& \Pi(\rho)=\left[\begin{array}{ccc}
\left\{-W(\rho) K \hat{D}_{a w}(\rho)-\hat{D}_{a w}^{T}(\rho) K W(\rho)\right. & -W(\rho) K D_{01}(\rho) & -\left(D_{10}(\rho)+D_{p 12}(\rho) \hat{D}_{a w}(\rho)\right)^{T} \\
+2 W(\rho) & \gamma I_{n_{d}} & -D_{11}^{T}(\rho) \\
-D_{01}^{T}(\rho) K W(\rho) & -D_{11}(\rho) & \gamma I_{n_{e}}
\end{array}\right]>0 \\
& {\left[\begin{array}{c|ccc}
0 & I_{n_{u}} & 0 & 0 \\
\hline I_{n_{u}} & & \\
0 & -\Pi(\rho) \\
0 & &
\end{array}\right]\left[\begin{array}{c}
\hat{B}_{a w}^{T}(\rho) \\
? ?
\end{array}\right]=-\left[\begin{array}{c}
0_{n_{u} \times n} \\
\hline B_{0}^{T}(\rho) S(\rho)+W(\rho) K C_{0}(\rho) \\
B_{1}^{T}(\rho) S(\rho) \\
C_{1}(\rho)
\end{array}\right]} \\
& {\left[\begin{array}{c|ccc}
0 & K W(\rho) & 0 & D_{p 12}^{T}(\rho) \\
\hline W(\rho) K & & \\
0 & -\Pi(\rho)
\end{array}\right]\left[\begin{array}{c}
\hat{C}_{a w}(\rho) \\
? ?
\end{array}\right]=-\left[\begin{array}{c}
B_{p 2}^{T}(\rho) \\
D_{p 12}(\rho)
\end{array}\right.} \\
& \hat{A}_{a w}(\rho, \dot{\rho})=-\dot{\rho} \frac{d S}{d \rho} R(\rho) H-\dot{\rho} \frac{d N}{d \rho} M^{T}(\rho) H-A^{T}(\rho, \dot{\rho}) H \\
& -\left[S(\rho) B_{0}(\rho)+\hat{B}_{a w}(\rho)+C_{0}^{T}(\rho) K W(\rho) \quad S(\rho) B_{1}(\rho) \quad C_{1}^{T}(\rho)\right] \Pi^{-1}(\rho) \\
& \times\left[\begin{array}{c}
\left(B_{0}^{T}(\rho)+W(\rho) K C_{0}(\rho) R(\rho)\right) H+\hat{D}_{a w}^{T}(\rho) B_{p 2}^{T}(\rho)+W(\rho) K \hat{C}_{a w}(\rho) \\
B_{1}^{T}(\rho) H \\
C_{1}(\rho) R(\rho) H+D_{p 12}(\rho) \hat{C}_{a w}(\rho)
\end{array}\right] \\
& {\left[\begin{array}{ll}
A_{a w}(\rho, \dot{\rho}) & B_{a w}(\rho) \\
C_{a w}(\rho, \dot{\rho}) & D_{a w}(\rho)
\end{array}\right]=\left[\begin{array}{cc}
N(\rho) & S(\rho) B_{2}(\rho) \\
0 & {\left[\begin{array}{ll}
0 & I_{n_{u}}
\end{array}\right]}
\end{array}\right]^{-1}\left(\left[\begin{array}{cc}
\hat{A}_{a w}(\rho, \dot{\rho}) & \hat{B}_{a w}(\rho) \\
\hat{C}_{a w}(\rho) & \hat{D}_{a w}(\rho)
\end{array}\right]-\left[\begin{array}{cc}
S(\rho) A(\rho, \dot{\rho}) R(\rho) H & 0 \\
0 & 0_{n_{u} \times n_{u}}
\end{array}\right]\right)} \\
& \times\left[\begin{array}{cc}
M^{T}(\rho) H & 0 \\
0 & I_{n_{u}}
\end{array}\right]^{-1}
\end{aligned}
$$

The F-16 is powered by an afterburning turbofan jet engine, which produces a thrust force in the $x$ axis. The LaRC data include a model of the engine in which the thrust response is modeled with a first-order lag; the lag time constant is a function of the actual engine power level and the commanded power. The throttle position is related to the commanded power level. For convenience, the actual power level is also considered as a state variable in longitudinal dynamics.

The state and input variables of the F-16 model are as follows:

$$
\begin{aligned}
& x= \begin{cases}V & \text { true airspeed }(\mathrm{ft} / \mathrm{s}) \\
\alpha & \text { angle-of-attack }(\mathrm{rad}) \\
q & \text { pitch rate }(\mathrm{rad} / \mathrm{s}) \\
\theta & \text { pitch angle }(\mathrm{rad}) \\
\text { pow } & \text { actual power level }(0-100)\end{cases} \\
& u= \begin{cases}\delta_{t h} & \text { throttle position }(0-1) \\
\delta_{e} & \text { elevator angle }(\mathrm{deg})(-25 d e g-25 d e g)\end{cases}
\end{aligned}
$$

By slight abuse of notations, the variables just listed also represent perturbations from their equilibrium states when linearization is considered. In addition, $V$, $q$, and flight-path angle $\gamma$ are selected as outputs. To describe the nonlinear F-16 model by an LPV system, we first need to find the wings-level equilibrium solutions at several flight conditions in the design envelope. The local linear models are then obtained by linearizing the nonlinear equations of motion at those equilibrium points. The flight envelope of interest covers aircraft speeds between 160 and $200 \mathrm{ft} / \mathrm{s}$ and angles-ofattack between 20 and $45 \mathrm{deg}$. These two variables are used as scheduling parameters in the LPV modeling of F-16 longitudinal dynamics. The points at which the nonlinear model is linearized are marked by a $\times$ symbol in Fig. 3. This group of linearized models consist of the LPV representation of the nonlinear F-16 longitudinal dynamics within the chosen flight envelope. The corresponding dynamic pressure range is between 20 and $50 \mathrm{lb} / \mathrm{ft}^{2}$. This portion of flight envelope is chosen because the moderately high angle-of-attack and the low dynamic pressure cause aerodynamic control 
surface saturation, which is a major concern in this research. The actuators are explicitly constrained. Most of the time, we found that the elevator angle saturation becomes a limiting factor for flight control effectiveness.

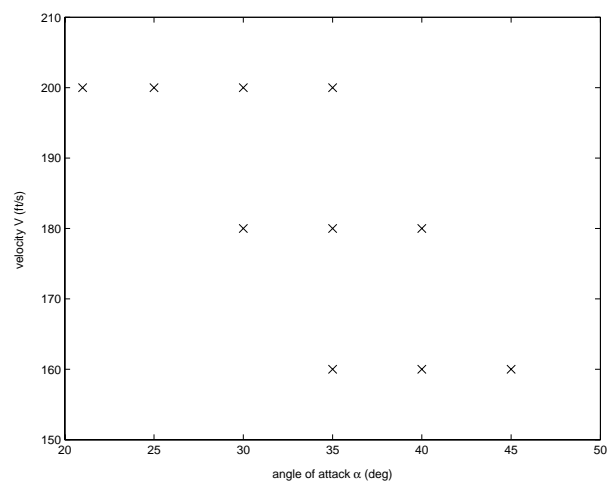

Fig. 3 Flight trim points.

\section{B. Problem Setup}

The design objective of the nominal LPV controller in this research is to track the flight-path angle command with the tracking error about $1.25 \%$ of the command in the steady-state motion. This kind of problem is conveniently formulated as a model-following problem, ${ }^{21}, 22$ where the ideal model to be followed is chosen to be a second-order filter based on desired flying qualities

$$
\frac{\gamma_{\text {ideal }}}{\gamma_{\text {cmd }}}=\frac{\omega_{\text {ideal }}^{2}}{s^{2}+2 \zeta_{\text {ideal }} \cdot \omega_{\text {ideal }} \cdot s+\omega_{\text {ideal }}^{2}}
$$

The implicit model-following framework allows for direct incorporation of flying quality specifications into the control design. A block diagram of the system interconnection for synthesizing the nominal controller is shown in Fig. 4, where $P_{\rho}$ is the model set of linearized aircraft dynamics at different operating points $\rho$ and $n$ is 3 -dimensional sensor noise.

The weighting functions are chosen as

$$
\begin{aligned}
W_{p} & =\frac{80(s / 5+1)}{s / 0.05+1}, \quad W_{n}=\operatorname{diag}\{0.8,0.6,0.1\} \\
W_{u} & =\operatorname{diag}\left\{1,10, \frac{1}{50}, \frac{1}{120}\right\} \\
W_{\text {ideal }} & =\frac{2.25}{s^{2}+2.4 s+2.25}
\end{aligned}
$$

The throttle and elevator actuator dynamics are modeled as first-order lag filters.

$$
\delta_{t h} / \delta_{t h}^{\mathrm{cmd}}=\frac{5}{s+5}, \quad \delta_{e} / \delta_{e}^{\mathrm{cmd}}=\frac{20}{s+20} .
$$

Both the positions and the rates of control inputs are fed into $W_{u}$ to penalize the control effort. Therefore, the system matrix of $W_{\text {act }}$ is derived as follows.

$$
W_{\text {act }}=\operatorname{diag}\left\{\left[\begin{array}{c|c}
-5 & 5 \\
\hline 1 & 0 \\
-5 & 5
\end{array}\right],\left[\begin{array}{c|c}
-20 & 20 \\
\hline 1 & 0 \\
-20 & 20
\end{array}\right]\right\}
$$

\section{Design Results}

The nominal controller is designed by formulating an LPV synthesis problem, which can be solved using either a single or parameter-dependent quadratic Lyapunov function over all gridding points in the two dimensional parameter space. ${ }^{14,15}$ In this work, the single quadratic Lyapunov function (SQLF) approach is chosen to reduce the computation time in the nominal LPV control synthesis phase. The performance obtained through the SQLF approach is $\gamma_{\text {nom }}=8.0$.

The proposed LPV antiwindup scheme is then applied to the control of an F-16 aircraft, and we also choose the SQLF approach to perform the LPV antiwindup synthesis. A series of LPV antiwindup compensators $A W_{\rho}$ are designed by gradually decreasing the $k_{i}(i=1,2)$ value from 1 to 0.99 . Table 1 shows the corresponding performance level $\gamma$ and the trade-off between the sector-bound constraint and the achieved performance. Because the linearized model at one of the gridding points is unstable, the synthesis condition for LPV antiwindup control is infeasible for $k_{i}=1$. Compared with the nominal performance, the worst performance level when $k_{i}=1-10^{-5}$ indicates the strong adverse effect of saturation nonlinearity. On the other hand, the antiwindup compensator almost recovers the nominal closed-loop performance $\gamma_{\text {nom }}$ when $k_{i}=0.99$. The antiwindup compensator corresponding to $k_{i}=0.999$ will be used in the following nonlinear simulations. The achieved performance by this LPV antiwindup compensator is 25.5 compared to the nominal performance of 8.0. Therefore the controlled performance will be sacrificed in order to achieve a guaranteed stability result.

Table $1 \quad \mathcal{H}_{\infty}$ performance level vs. sector range $\left[0, k_{i}\right]$.

\begin{tabular}{cc}
\hline \hline Sector range $\left[0, k_{i}\right]$ & $\mathcal{H}_{\infty}$ performance $\gamma$ \\
\hline $1-10^{-5}$ & 1251.92 \\
$1-10^{-4}$ & 196.55 \\
0.999 & 25.50 \\
0.99 & 8.00 \\
\hline \hline
\end{tabular}

\section{Nonlinear Simulations}

The LPV nominal controller $K_{\rho}$ and antiwindup compensator $A W_{\rho}$ are tested first at one designed flight condition, which is a trimmed flight at $V=$ $160 \mathrm{ft} / \mathrm{s}$ and $\alpha=35 \mathrm{deg}$. The magnitude limits of actuators are enforced during the nonlinear simulation. A flight-path doublet input with magnitude $\pm 2 d e g$ is used to demonstrate the performance of the antiwindup compensator. Both the nominal controller and antiwindup compensator are fixed LTI controllers for the given flight condition derived from the LPV controllers $K_{\rho}$ and $A W_{\rho}$. The responses of the nonlinear system are shown in Fig. 5, where the solid line is the trajectory with the antiwindup compensator, and the 


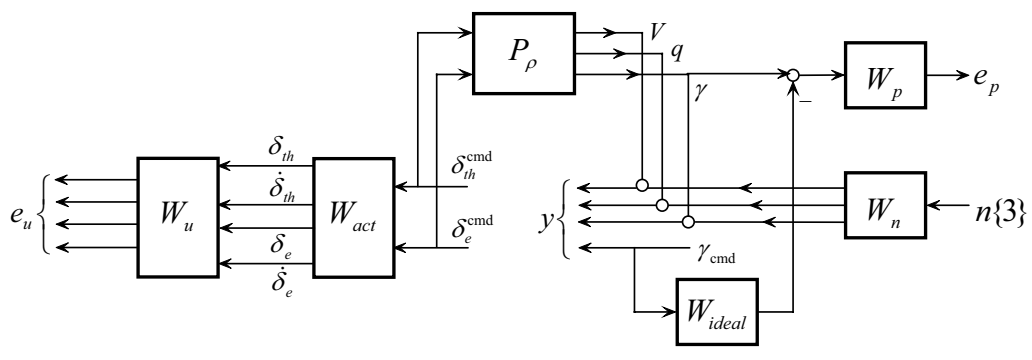

Fig. 4 Open-loop interconnection for LPV control design.

dashed line is the trajectory without the antiwindup compensator and controlled only by the nominal controller. The dotted line in Fig. 5 (a) represents the flight-path angle response of the ideal model. It is observed that the performance without the antiwindup compensator becomes worse around $t=15 \mathrm{~s}$ because of elevator saturation. However, the insertion of the antiwindup compensator quickly overcomes the saturation and greatly improves the tracking performance.

Another test condition is selected at $V=200 \mathrm{ft} / \mathrm{s}$ and $\alpha=22 d e g$, which is not a designed flight condition, so both the nominal controller and antiwindup compensator are not fixed LTI controllers but varying in time. However, the computation time will be increased greatly if the controllers are constructed online according to the current parameter value. To reduce computational cost, a relatively coarse gridding parameter space $\mathcal{P}$ is used for synthesis, and then $K_{\rho}$ and $A W_{\rho}$ are constructed off-line at finer gridding points and saved as look-up tables. In our study, 10 points are used for synthesis as shown in Fig. 3, and 45 points for constructing look-up tables of $K_{\rho}$ and $A W_{\rho}$. When doing simulations, the nominal controller and antiwindup compensator gains are calculated using linear interpolation at the current values of the scheduling parameters between the grid point solutions.

Note that the nominal controller $K_{\rho}$ is designed for a set of linearized plant models at the gridding points, so that the output of $K_{\rho}$ is the control deviation from the nominal control maintaining trim condition, and the actual control input to the nonlinear plant should be the summation of both terms. When $K_{\rho}$ is parameter varying in simulations, the instant nominal control inputs should be determined at each time. This can be done by building up another look-up table of nominal control inputs at finer gridding points beforehand and interpolating in real time according to the current parameter values.

For the flight condition just listed, a flight-path doublet input with magnitude $\pm 4 d e g$ is used. The simulation results has confirmed that the trajectory of parameters evolves about half of the parameter space. In this case, the system might not achieve the desired performance, even maintain stability by using a single
LTI antiwindup compensator. Instead, it is crucial to use LPV nominal control and LPV antiwindup compensation to achieve good controlled performance. It is also shown that the variations of the scheduling parameters do not exceed the assumed limits: $|\dot{V}| \leq 20 \mathrm{ft} / \mathrm{s}^{2}$ and $|\dot{\alpha}| \leq 10 \mathrm{deg} / \mathrm{s}$, which are used in the LPV control synthesis stage. Moreover, the sector bound constraint on control input $u_{i} \leq 1000 u_{i}^{\max }$ is satisfied over the entire simulation time. Fig. 6 shows the aircraft response with an LPV antiwindup compensator, and the dotted line in Fig. 6(a) is the ideal flight-path angle response. It is observed that the LPV antiwindup compensator achieves the desired performance objective. However, when the antiwindup compensator is unused, both the elevator and the throttle are saturated severely and the system goes unstable. For clarity purpose, the response without LPV antiwindup compensator will not be shown in Fig. 6. Note that the variations of elevator angle and throttle position in this case study might go beyond the typical rate saturation limits, and thus are not realistic for implementation.

\section{E. Comparison with Thrust Vectoring Control}

Unconventional control effectors like thrust vectoring are another way to enhance maneuverability of the modern aircraft at and beyond the stall angle-ofattack. To compare with the saturation control scheme using an antiwindup compensator, a simple thrust vectoring model is added to the previous nonlinear F-16 model to provide additional longitudinal axis control power. For simplicity, there are only thrust components along $x$ and $z$ body axes. A detailed description of the thrust vectoring model can be found in Ref. 23 .

Now the input variables are elevator deflection $\delta_{e}$ and pitch thrust vectoring nozzle deflection $\delta_{p t v}$, whose position limit is $-17 \mathrm{deg} \leq \delta_{p t v} \leq 17 \mathrm{deg}$. Because the state variable pow is not related to either input in the linearized model, it is neglected and the number of states is reduced to four. The longitudinal linear model of the F-16 aircraft at a given trim point with throttle and elevator as controls and that with elevator and thrust vectoring as controls are

$$
\dot{x_{1}}=A_{1} x_{1}+B_{1} u_{1}=A_{1} x_{1}+B_{1}\left[\begin{array}{c}
\delta_{t h} \\
\delta_{e}
\end{array}\right]
$$




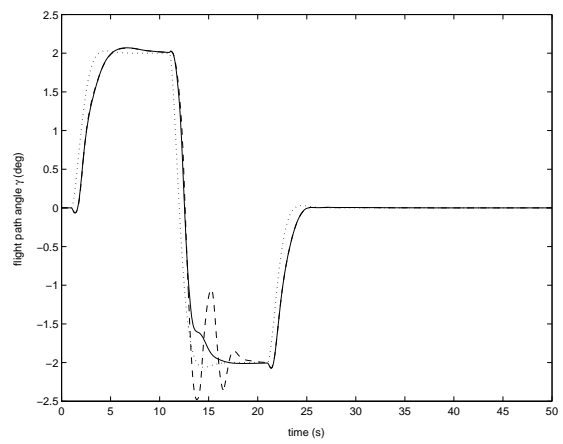

(a) Flight-path angle

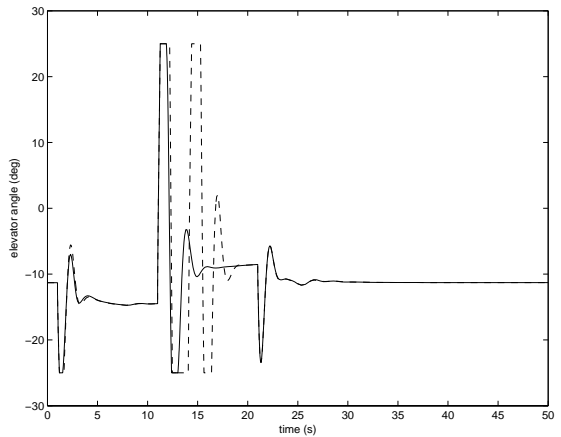

(c) Elevator angle

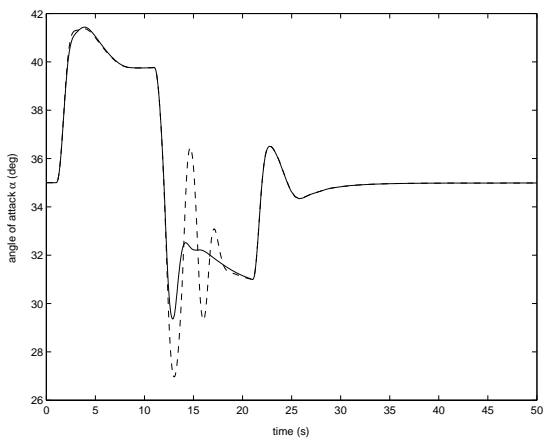

(b) Angle-of-attack

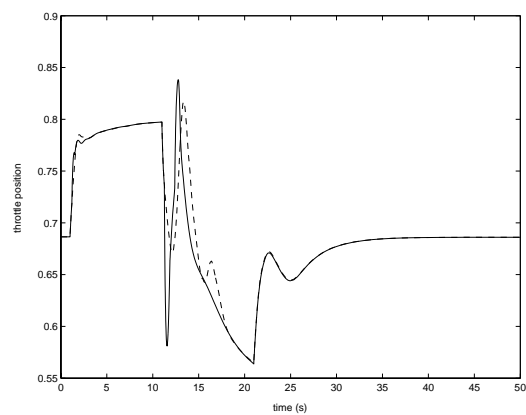

(d) Throttle position

Fig. 5 Saturation control of F-16 dynamics with and without LTI antiwindup compensator.

$$
\dot{x_{2}}=A_{2} x_{2}+B_{2} u_{2}=A_{2} x_{2}+B_{2}\left[\begin{array}{c}
\delta_{p t v} \\
\delta_{e}
\end{array}\right]
$$

where the states $x_{2}$ include velocity $V$, angle-of-attack $\alpha$, pitch rate $q$, and pitch angle $\theta$.

There are usually two methods to generate thrust vector commands, daisy-chain ${ }^{21,23}$ and ganged control methods., 24,25 For both methods, a controller is designed first based on the generalized control, and the real control inputs are then generated using a control selector. The former approach commands thrust vectoring only when the conventional aerodynamic control surfaces are ineffective, i.e. they are unable to generate the necessary forces and moments required for commanded maneuvers. However, it is difficult to define the generalized control for high-order systems. The basic idea of the latter approach is to redefine the control contribution to the state dynamics equation with different weights, and the thrust vectoring always works in flight control. The disadvantage of the ganged control scheme is that it cannot precisely determine the weights of different controls.

The control allocation method used in this research is different from those two approaches because the generalized control to design the controller is not used. The aircraft model with conventional control effectors is used in the stage of controller design and the designed controller is considered as the nominal controller $K_{\rho}$. A control allocation function is then implemented in the nonlinear simulation and thrust vectoring is commanded only when the elevator un- dergoes saturation. So, the thrust vectoring control is not used for control design and only used for control implementation. This control allocation method avoids defining the generalized control and setting the weights for different control effectors.

In the linearized model with conventional control effectors (21), the state variable pow is only related to the throttle, which has no relation with the four other states. For simplicity in describing the control allocation scheme, the first four states with only elevator deflection are considered in (21). So the states and $A$ matrix of the two linearized models are the same.

$$
\begin{aligned}
& \dot{x}=A x+B_{1}^{*} u_{1}^{*}=A x+B_{1}^{*} \delta_{e}^{*} \\
& \dot{x}=A x+B_{2} u_{2}=A x+B_{2}\left[\begin{array}{c}
\delta_{p t v} \\
\delta_{e}
\end{array}\right]
\end{aligned}
$$

where $B_{1}^{*}$ represents the simplified input matrix, and $\delta_{e}^{*}$ is the elevator deflection. Ideally, we would like the actual control

$$
B_{2}\left[\begin{array}{c}
\delta_{p t v} \\
\delta_{e}
\end{array}\right]
$$

to provide control effect equivalent to that of the artificial control $B_{1}^{*} \delta_{e}^{*}$. Then the practical thrust vectoring and elevator angle can be obtained by solving the following optimization problem when the elevator is saturated.

$$
\min \left\|B_{2} u_{2}-B_{1}^{*} u_{1}^{*}\right\|_{2}
$$

subj. to $\left[\begin{array}{c}-17+\delta_{p t v}^{0} \\ -25+\delta_{e}^{0}\end{array}\right] \leq\left[\begin{array}{c}\delta_{p t v} \\ \delta_{e}\end{array}\right] \leq\left[\begin{array}{c}17+\delta_{p t v}^{0} \\ 25+\delta_{e}^{0}\end{array}\right]$ 


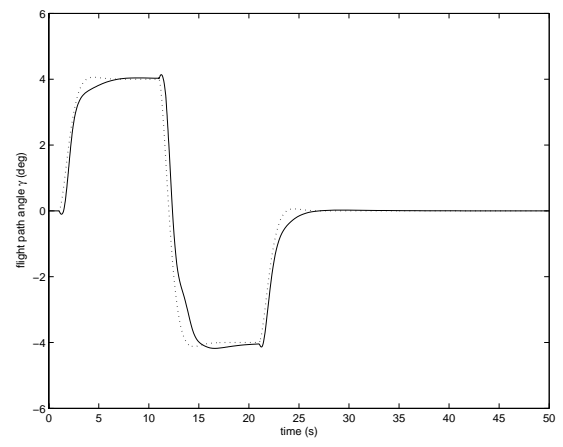

(a) Flight-path angle

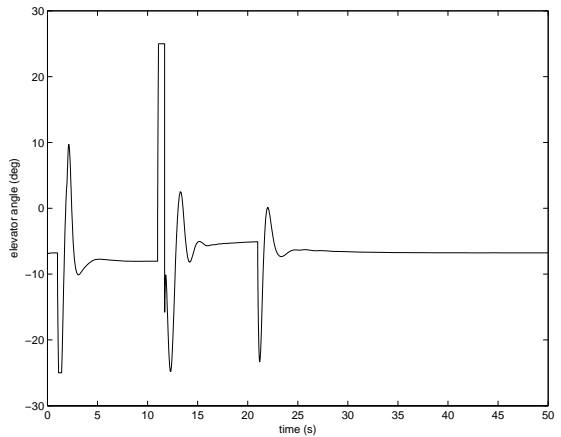

(c) Elevator angle

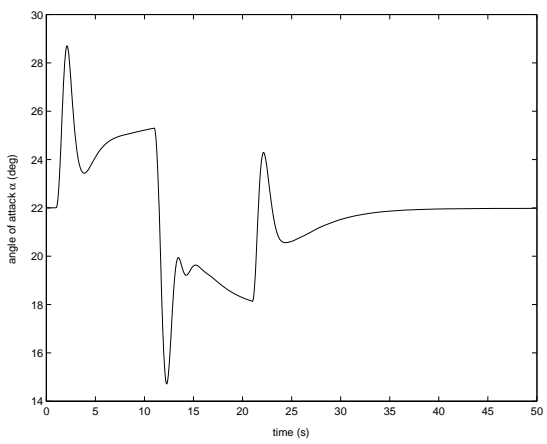

(b) Angle-of-attack

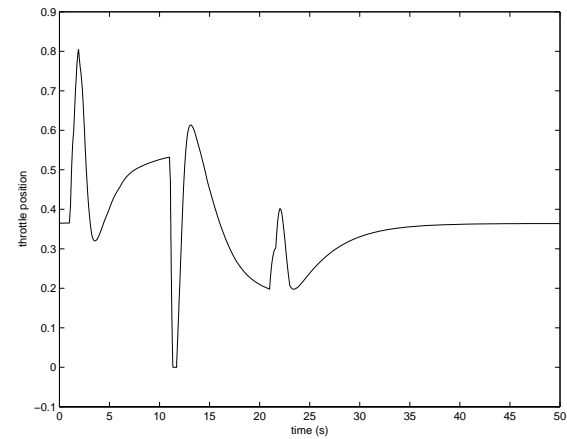

(d) Throttle position

Fig. 6 Nonlinear doublet response with LPV antiwindup compensator.

where $\delta_{p t v}^{0}$ and $\delta_{e}^{0}$ are equilibrium inputs at trim condition.

To compare the control effect of the antiwindup compensator and thrust vectoring, a \pm 2 deg flight path doublet is commanded at the flight condition $V=$ $160 \mathrm{ft} / \mathrm{s}$ and $\alpha=35 \mathrm{deg}$, same as in Fig. 5. The solid line in Fig. 7 represents the response of the closed-loop system with thrust vectoring. Similarly, the dotted line in Fig. 7 is the ideal trajectory of the flight-path angle. A comparison between the solid responses in Figs. 5 and 7 illustrates that the antiwindup control achieves better tracking performance than thrust vectoring control scheme.

\section{Conclusion}

The goal of flight control in the high angle-of-attack region is to maintain aircraft stability with acceptable flight qualities. However, the saturation of the conventional aerodynamic control surfaces presents many challenges to the control designers, for example, actuator saturation is an important issue to near-stall and poststall flight conditions. Without cost/hardware concerns, unconventional actuators such as thrust vectoring are usually suggested to compensate the conventional aerodynamic control surfaces. However, the incorporation of additional thrust vectoring nozzle could complicate the design of flight control laws in the poststall regime. The antiwindup method provides an alternative approach to handle control saturation. Applying antiwindup control scheme to flight control is promising because no additional actuator is needed to compensate control authority. The implementation of antiwindup controllers could be done by simply modifying flight control software.

An antiwindup control scheme for LPV systems has been developed in this research. The extension of the antiwindup control idea to LPV systems provides a practical approach for nonlinear flight dynamics in the presence of actuator saturation. Because of the special structure of the antiwindup control scheme, the LPV antiwindup control synthesis condition is solvable by LMI optimizations. The LPV antiwindup compensation has been applied to an F-16 aircraft and compared with the thrust vectoring control scheme. By augmenting the nominal longitudinal autopilot with an antiwindup compensator, it has been shown through nonlinear simulation that the F-16 aircraft maintains stability and adequate control performance in case of actuator saturation, while the performance achieved by the thrust vectoring control scheme is undesirable when a large maneuvering operation is commanded.

\section{Appendix: Proof of Main Results}

\section{Theorem 1}

Proof: Consider a Lyapunov function $V(x)=$ $x^{T} X_{c l}(\rho) x$ for the closed-loop system $T$, then a sufficient condition for the exponential stability and performance can be established from the inequality

$$
\dot{V}+\frac{1}{\gamma} e^{T} e-\gamma d^{T} d+2 q^{T} W(K u-q)<0
$$




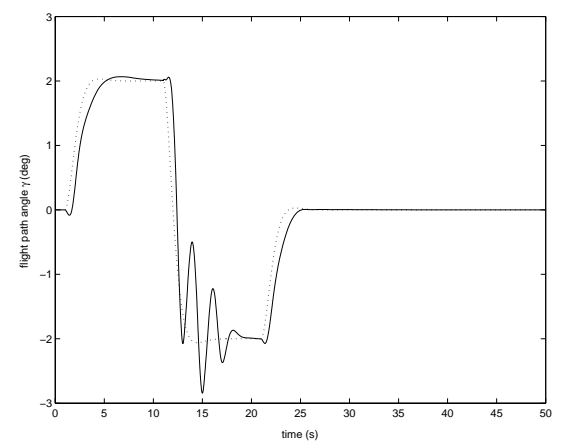

(a) Flight-path angle

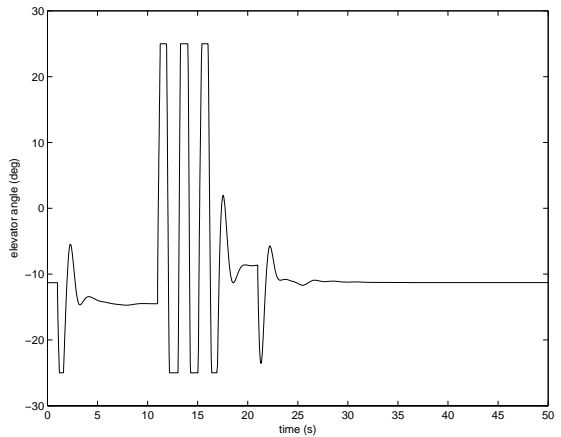

(c) Elevator angle

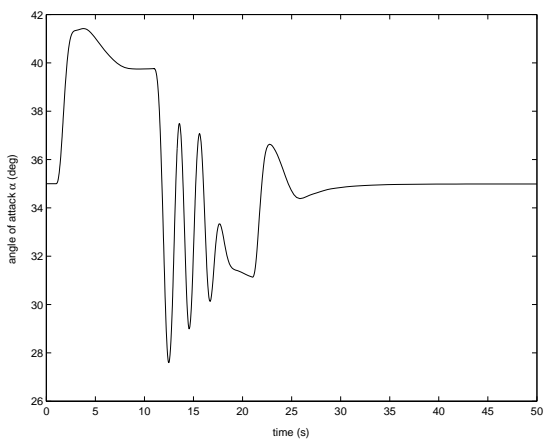

(b) Angle-of-attack

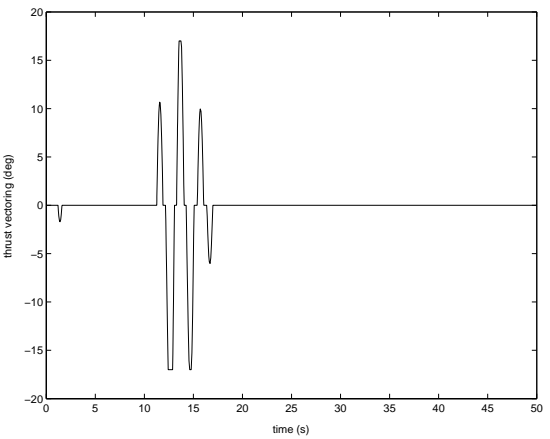

(d) Thrust vectoring nozzle deflection

Fig. 7 Nonlinear doublet response with thrust vectoring.

using $S$-procedure. Note that it is equivalent to

$$
\begin{aligned}
& {\left[\begin{array}{cc}
\left\{\begin{array}{c}
A_{c l}^{T} X_{c l}+X_{c l} A_{c l} \\
+\sum_{i=1}^{s} \dot{\rho}_{i} \frac{\partial X_{c l}}{\partial \rho_{i}}
\end{array}\right\} & X_{c l} B_{0, c l}+C_{0, c l}^{T} K W \\
B_{0, c l}^{T} X_{c l}+W K C_{0, c l} & \left\{W K D_{00, c l}+D_{00, c l}^{T} K W\right. \\
-2 W & D_{01, c l}^{T} K W \\
B_{1, c l}^{T} X_{c l} & D_{10, c l} \\
C_{1, c l} &
\end{array}\right.} \\
& \left.\begin{array}{cc}
X_{c l} B_{1, c l} & C_{1, c l}^{T} \\
W K D_{01, c l} & D_{10, c l}^{T} \\
-\gamma I & D_{11, c l}^{T} \\
D_{11, c l} & -\gamma I
\end{array}\right]<0
\end{aligned}
$$

where the closed-loop state-space data are as follows: where

$$
\begin{aligned}
& \mathcal{A}(\rho, \dot{\rho})=\left[\begin{array}{cc}
A(\rho, \dot{\rho}) & 0 \\
0 & 0
\end{array}\right] \\
& \mathcal{B}_{0}(\rho)=\left[\begin{array}{c}
B_{0}(\rho) \\
0
\end{array}\right], \quad \mathcal{B}_{1}(\rho)=\left[\begin{array}{c}
B_{1}(\rho) \\
0
\end{array}\right] \\
& \mathcal{C}_{0}(\rho)=\left[\begin{array}{ll}
C_{0}(\rho) & 0
\end{array}\right], \quad \mathcal{C}_{1}(\rho)=\left[\begin{array}{ll}
C_{1}(\rho) & 0
\end{array}\right] \\
& {\left[\begin{array}{c}
\mathcal{P}_{1}^{T}(\rho) \\
\mathcal{P}_{2}^{T}(\rho) \\
\mathcal{P}_{3}^{T}(\rho)
\end{array}\right]=\left[\begin{array}{cc}
0 & B_{2}(\rho) \\
I & 0 \\
\hline 0 & D_{02}(\rho) \\
\hline 0 & D_{12}(\rho)
\end{array}\right]} \\
& {\left[\begin{array}{lll}
\mathcal{Q}_{1} & \mathcal{Q}_{2} & \mathcal{Q}_{3}
\end{array}\right]=\left[\begin{array}{ll|l|l}
0 & I & 0 & 0 \\
0 & 0 & I & 0
\end{array}\right]}
\end{aligned}
$$

$$
\begin{gathered}
{\left[\begin{array}{ccc}
A_{c l}(\rho, \dot{\rho}) & B_{0, c l}(\rho) & B_{1, c l}(\rho) \\
C_{0, c l}(\rho, \dot{\rho}) & D_{00, c l}(\rho) & D_{01, c l}(\rho) \\
C_{1, c l}(\rho, \dot{\rho}) & D_{10, c l}(\rho) & D_{11, c l}(\rho)
\end{array}\right]} \\
=\left[\begin{array}{ccc}
\mathcal{A}(\rho, \dot{\rho}) & \mathcal{B}_{0}(\rho) & \mathcal{B}_{1}(\rho) \\
\mathcal{C}_{0}(\rho) & D_{00}(\rho) & D_{01}(\rho) \\
\mathcal{C}_{1}(\rho) & D_{10}(\rho) & D_{11}(\rho)
\end{array}\right]+\left[\begin{array}{ll}
\mathcal{P}_{1}^{T}(\rho) \\
\mathcal{P}_{2}^{T}(\rho) \\
\mathcal{P}_{3}^{T}(\rho)
\end{array}\right] \\
\quad \times\left[\begin{array}{lll}
A_{a w}(\rho, \dot{\rho}) & B_{a w}(\rho) \\
C_{a w}(\rho, \dot{\rho}) & D_{a w}(\rho)
\end{array}\right]\left[\begin{array}{lll}
\mathcal{Q}_{1} & \mathcal{Q}_{2} & \mathcal{Q}_{3}
\end{array}\right]
\end{gathered}
$$

Denote $\Theta(\rho, \dot{\rho})=\left[\begin{array}{ll}A_{a w}(\rho, \dot{\rho}) & B_{a w}(\rho) \\ C_{a w}(\rho, \dot{\rho}) & D_{a w}(\rho)\end{array}\right]$ and $V(\rho)=$ $W^{-1}(\rho)$. Inequality (A1) can be rewritten as follows:$$
\Psi(\rho, \dot{\rho})+\mathcal{P}^{T}(\rho) \Theta(\rho, \dot{\rho}) \mathcal{Q}+\mathcal{Q}^{T} \Theta^{T}(\rho, \dot{\rho}) \mathcal{P}(\rho)<0
$$ 
for all $(\rho, \dot{\rho}) \in \mathcal{P} \times \mathcal{V}$ with

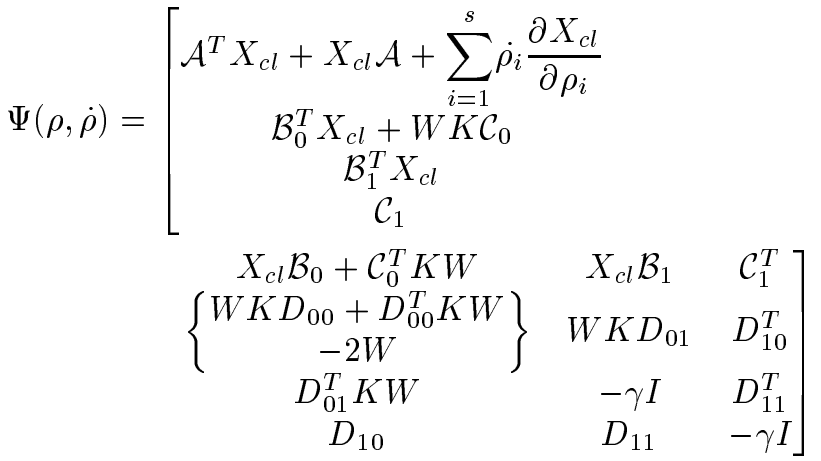

$$
\begin{aligned}
& \mathcal{P}(\rho)=\left[\begin{array}{llll}
\mathcal{P}_{1} X_{c l} & \mathcal{P}_{2} K W & 0 & \mathcal{P}_{3}
\end{array}\right] \\
& \mathcal{Q}=\left[\begin{array}{llll}
\mathcal{Q}_{1} & \mathcal{Q}_{2} & \mathcal{Q}_{3} & 0
\end{array}\right]
\end{aligned}
$$

Partition the matrix function $X_{c l}$ compatibly to the states of an interconnected system $G_{\rho}$ and antiwindup compensator $A W_{\rho}$ as $n=n_{p}+n_{k}$ and $n_{a w}$, and let

$$
\begin{aligned}
X_{c l}(\rho) & =\left[\begin{array}{cc}
S(\rho) & N(\rho) \\
N^{T}(\rho) & ?
\end{array}\right] \\
X_{c l}^{-1}(\rho) & =\left[\begin{array}{cc}
R(\rho) & M(\rho) \\
M^{T}(\rho) & ?
\end{array}\right] \\
& =\left[\begin{array}{cc|c}
R_{11}(\rho) & R_{12}(\rho) & M(\rho) \\
R_{12}^{T}(\rho) & R_{22}(\rho) & \\
\hline M^{T}(\rho) & ?
\end{array}\right]
\end{aligned}
$$

where $M(\rho) N^{T}(\rho)=I-R(\rho) S(\rho)$. According to elimination lemma, ${ }^{26}$ inequality (A3) is equivalent to

$$
\mathcal{N}_{\mathcal{P}}^{T}(\rho) \Psi(\rho, \dot{\rho}) \mathcal{N}_{\mathcal{P}}(\rho)<0 \quad \text { and } \quad \mathcal{N}_{\mathcal{Q}}^{T} \Psi(\rho, \dot{\rho}) \mathcal{N}_{\mathcal{Q}}<0
$$

for all $(\rho, \dot{\rho}) \in \mathcal{P} \times \mathcal{V} . \mathcal{N}_{\mathcal{P}}$ and $\mathcal{N}_{\mathcal{Q}}$ expand the null spaces of matrix functions $\mathcal{P}$ and $\mathcal{Q}$, which are

$$
\begin{aligned}
& \mathcal{N}_{\mathcal{P}}(\rho)=\operatorname{diag}\left\{X_{c l}^{-1}(\rho), W^{-1}(\rho), I, I\right\} \\
& \times\left[\begin{array}{ccc}
I & 0 & 0 \\
0 & 0 & 0 \\
0 & 0 & 0 \\
\hline-K^{-1} B_{p 2}^{T}(\rho) & -K^{-1} D_{p 12}^{T}(\rho) & 0 \\
\hline 0 & 0 & I \\
\hline 0 & I & 0
\end{array}\right] \\
& \mathcal{N}_{\mathcal{Q}}=\left[\begin{array}{llll}
I & 0 & 0 & 0 \\
0 & I & 0 & 0 \\
0 & 0 & 0 & 0 \\
\hline 0 & 0 & 0 & 0 \\
\hline 0 & 0 & I & 0 \\
\hline 0 & 0 & 0 & I
\end{array}\right]
\end{aligned}
$$

Through lengthy algebraic manipulations, it can be shown that

$$
\begin{aligned}
& \mathcal{N}_{\mathcal{P}}^{T}(\rho) \Psi(\rho, \dot{\rho}) \mathcal{N}_{\mathcal{P}}(\rho) \\
& =\left[\begin{array}{c}
\left\{\begin{array}{c}
R_{11} A_{p}^{T}+A_{p} R_{11}-\sum_{i=1}^{s} \dot{\rho}_{i} \frac{\partial R_{11}}{\partial \rho_{i}} \\
+2 B_{p 2} K^{-1} V\left(I-K^{-1}\right) B_{p 2}^{T}
\end{array}\right\} \\
C_{p 1} R_{11} \\
+2 D_{p 12} K^{-1} V\left(I-K^{-1}\right) B_{p 2}^{T} \\
B_{p 1}^{T}
\end{array}\right\}
\end{aligned}
$$

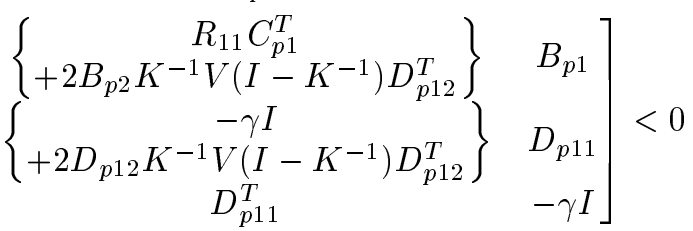

$\mathcal{N}_{\mathcal{Q}}^{T} \Psi(\rho, \dot{\rho}) \mathcal{N}_{\mathcal{Q}}$

$$
=\left[\begin{array}{ccc}
S A+A^{T} S+\sum_{i=1}^{s} \dot{\rho}_{i} \frac{\partial S}{\partial \rho_{i}} & S B_{1} & C_{1}^{T} \\
B_{1}^{T} S & -\gamma I & D_{11}^{T} \\
C_{1} & D_{11} & -\gamma I
\end{array}\right]<0
$$

which are the same as conditions (8) and (9), respectively.

Given the definition of matrix functions $X_{c l}$ and $X_{c l}^{-1}$, the coupling condition between $R$ and $S$ would be

$\left[\begin{array}{cc}R(\rho) & I \\ I & S(\rho)\end{array}\right] \geq 0 \quad$ and $\quad \operatorname{rank}\left[R(\rho)-S^{-1}(\rho)\right] \leq n_{a w}$

for all $\rho \in \mathcal{P}$. This is equivalent to the third LMI because only $R_{11}$ is constrained.

Q.E.D.

\section{Theorem 2}

Proof: The derivation of the antiwindup controller formula basically follows the procedure outlined in Ref. 12. Define

$$
Z_{1}=\left[\begin{array}{cc}
I & R H \\
0 & M^{T} H
\end{array}\right], \quad Z_{2}=\left[\begin{array}{cc}
S & H \\
N^{T} & 0
\end{array}\right]
$$


Then it can be shown that $X_{c l} Z_{1}=Z_{2}$. Also we have the following congruent transformation:

$$
\begin{aligned}
& Z_{1}^{T} X_{c l} Z_{1}=\left[\begin{array}{cc}
S & H \\
H^{T} & H^{T} R H
\end{array}\right] \\
& Z_{1}^{T} \frac{d X_{c l}}{d t} Z_{1} \\
& =\left[\begin{array}{cc}
\frac{d S}{d t} & \left(R \frac{d S}{d t}+M \frac{d N^{T}}{d t}\right)^{T} H \\
H^{T}\left(R \frac{d S}{d t}+M \frac{d N^{T}}{d t}\right) & -H^{T} \frac{d R}{d t} H
\end{array}\right] \\
& {\left[\begin{array}{ccc}
Z_{1}^{T} X_{c l} A_{c l} Z_{1} & Z_{1}^{T} X_{c l} B_{0, c l} & Z_{1}^{T} X_{c l} B_{1, c l} \\
C_{0, c l} Z_{1} & D_{00, c l} & D_{01, c l} \\
C_{1, c l} Z_{1} & D_{10, c l} & D_{11, c l}
\end{array}\right]} \\
& =\left[\begin{array}{cc|c|c}
S A & 0 & S B_{0} & S B_{1} \\
H^{T} A & H^{T} A R H & H^{T} B_{0} & H^{T} B_{1} \\
\hline C_{0} & C_{0} R H & D_{00} & D_{01} \\
\hline C_{1} & C_{1} R H & D_{10} & D_{11}
\end{array}\right] \\
& +\left[\begin{array}{cc}
I_{n} & 0 \\
0 & B_{p 2} \\
\hline 0 & I \\
\hline 0 & D_{p 12}
\end{array}\right]\left[\begin{array}{ll}
\hat{A}_{a w} & \hat{B}_{a w} \\
\hat{C}_{a w} & \hat{D}_{a w}
\end{array}\right]\left[\begin{array}{cc|c|c}
0 & I_{n_{p}} & 0 & 0 \\
0 & 0 & I & 0
\end{array}\right]
\end{aligned}
$$

where

$$
\begin{aligned}
& {\left[\begin{array}{cc}
\hat{A}_{a w} & \hat{B}_{a w} \\
\hat{C}_{a w} & \hat{D}_{a w}
\end{array}\right]=\left[\begin{array}{cc}
S A R H & 0 \\
0 & 0
\end{array}\right]} \\
& +\left[\begin{array}{cc}
N & S B_{2} \\
0 & {\left[\begin{array}{cc}
0 & I
\end{array}\right]}
\end{array}\right]\left[\begin{array}{ll}
A_{a w} & B_{a w} \\
C_{a w} & D_{a w}
\end{array}\right]\left[\begin{array}{cc}
M^{T} H & 0 \\
0 & I
\end{array}\right]
\end{aligned}
$$

Multiply $\operatorname{diag}\left\{Z_{1}^{T}, I, I, I\right\}$ from the left side and its conjugate transpose from the right side of eq. (A3) and we get

$$
\left[\begin{array}{cc|c}
J_{11} & J_{21}^{T} & L_{1}^{T} \\
J_{21} & J_{22} & L_{2}^{T} \\
\hline L_{1} & L_{2} & -\Pi
\end{array}\right]<0
$$

with the shorthand notation

$$
\begin{aligned}
J_{11}= & S A+A^{T} S+\sum_{i=1}^{s} \dot{\rho}_{i} \frac{\partial S}{\partial \rho_{i}} \\
J_{21}= & H^{T} A+\hat{A}_{a w}^{T}+H^{T}\left(R \frac{d S}{d t}+M \frac{d N^{T}}{d t}\right) \\
J_{22}= & H^{T}\left(A R+R A^{T}-\sum_{i=1}^{s} \dot{\rho}_{i} \frac{\partial R}{\partial \rho_{i}}\right) H \\
& +B_{p 2} \hat{C}_{a w}+\hat{C}_{a w}^{T} B_{p 2}^{T} \\
L_{1}= & {\left[\begin{array}{c}
B_{0}^{T} S+\hat{B}_{a w}^{T}+W K C_{0} \\
B_{1}^{T} S \\
C_{1}
\end{array}\right] } \\
L_{2}= & {\left[\begin{array}{c}
\left(B_{0}^{T}+W C_{0} R\right) E+\hat{D}_{a w}^{T} B_{p 2}^{T}+W K \hat{C}_{a w} \\
B_{1}^{T} H \\
C_{1} R H+D_{p 12} \hat{C}_{a w}
\end{array}\right] }
\end{aligned}
$$

By Schur complement, it is equivalent to

$$
\left[\begin{array}{ll}
J_{11}+L_{1}^{T} \Pi^{-1} L_{1} & J_{21}^{T}+L_{1}^{T} \Pi^{-1} L_{2} \\
J_{21}+L_{2}^{T} \Pi^{-1} L_{1} & J_{22}+L_{2}^{T} \Pi^{-1} L_{2}
\end{array}\right]<0
$$

Clearly, the lower $(3 \times 3)$ matrix of inequality $(\mathrm{A} 5)$ must be negative definite; this determines the feasible $\hat{D}_{a w}$. Let the $(2,1)$ element be equal to zeros and one can solve for $\hat{A}_{a w}$. This also leads to decoupled LMIs from the inequality (A6). Then $\hat{B}_{a w}, \hat{C}_{a w}$ terms can be solved from the $(1,1)$ and $(2,2)$ elements of the decoupled inequality (A6). Note that both inequalities have regular solutions. ${ }^{27}$

The $(1,1)$ element of the above matrix inequality corresponds to LMI (9) after elimination of the variables $\hat{B}_{a w}$ and $\hat{D}_{a w}$. It can also be shown that the $(2,2)$ element is equivalent to LMI $(8)$ by eliminating $\hat{C}_{a w}, \hat{D}_{a w}$.

Q.E.D.

\section{Acknowledgment}

The first two authors would like to acknowledge the financial support for this research by NASA Langley Research Center under grant NAG-1-01119.

\section{References}

${ }^{1}$ Reigelsperger, W.C., and Banda, S.S., "Nonlinear Simulation for A Modified F-16 with Full-Envelope Control Laws," Control Engineering Practice, Vol. 6, No. 3, 1998, pp. 309-320.

${ }^{2}$ Balas, G.J., Doyle, J.C., Glover, K., Packard, A.K., and Smith, R., $\mu$-Analysis and Synthesis Toolbox, Mathworks Inc., Natick, MA, 1991.

${ }^{3}$ Adams, R.J., Buffington, J.M., Sparks, A.G., and Banda, S.S., Robust Multivariable Flight Control, Springer-Verlag, London, UK, 1994.

${ }^{4}$ Dornheim, M.A., "Report Pinpoints Factors Leading to YF-22 Crash," Aviation Week Space Technol., Nov. 9, 1992, pp. 53-54.

${ }^{5}$ Khalil, H.K., Nonlinear Systems, 2nd ed., Prentice-Hall, Englewood Cliffs, NJ, 1996.

${ }^{6}$ Kothare, M.V., Campo, P.J., Morari, M., and Nett, C.N., "A Unified Framework for The Anti-Windup Designs," Automatica, Vol. 30, No. 12, 1994, pp. 1869-1883.

${ }^{7}$ Mulder, E.F., Kothare, M.V., and Morari, M., "Multivariable Anti-Windup Controller Synthesis Using Linear Matrix Inequalities," Automatica, Vol. 37, No. 9, 2001, pp. 1407-1416.

${ }^{8}$ Grimm, G., Hatfield, J., Postlethwaite, I., Teel, A.R., Turner, M.C., and Zaccarian, L., "Antiwindup for Stable Linear Systems with Input Saturation: An LMI-Based Synthesis," IEEE Transactions on Automatic Control, Vol. 48, No. 9, 2003, pp. $1509-1525$.

${ }^{9}$ Tyan, F., and Bernstein, D.S., "Anti-Windup Compensator Synthesis for Systems with Saturation Actuators," International Journal of Robust and Nonlinear Control, Vol. 5, 1995, pp. 521-537.

${ }^{10}$ Teel, A.R., "Anti-Windup for Exponentially Unstable Linear Systems," International Journal of Robust and Nonlinear Control, Vol. 9, No. 10, 1999, pp. 701-716.

${ }^{11} \mathrm{Hu}, \mathrm{T}$., and Lin, Z., Control Systems with Actuator Saturation: Analysis and Design, Birkhäuser, Boston, 2001.

${ }^{12} \mathrm{Wu}, \mathrm{F}$., and Lu, B., "Anti-Windup Control Design for Exponentially Unstable LTI Systems with Actuator Saturation," Proceedings of 2003 American Control Conference, Denver, Colorado, June 2003, pp. 343-347.

${ }^{13} \mathrm{Wu}, \mathrm{F}$., and Soto, M., "Extended Anti-Windup Control of LTI Systems with Actuator Magnitude and Rate Saturations," Proceedings of 42nd IEEE Conference on Decision and Control, Maui, Hawaii, Dec. 2003, pp. 2786-2791. 
${ }^{14}$ Becker, G., and Packard, A., "Robust Performance of Linear Parametrically Varying Systems Using ParametricallyDependent Linear Feedback," Systems \& Control Letters, Vol. 23, No. 3, 1994, pp. 205-215.

${ }^{15} \mathrm{Wu}$, F., Yang, X.H., Packard, A., and Becker, G., "Induced $\mathcal{L}_{2}$ Norm Control for LPV Systems with Bounded Parameter Variation Rates," International Journal of Robust and Nonlinear Control, Vol. 6, No. 9-10, 1996, pp. 983-998.

${ }^{16}$ Apkarian, P., and Adams, R.J., "Advanced GainScheduling Techniques for Uncertain System," IEEE Transactions on Control Systems Technology, Vol. 6, No. 1, 1998, pp. 21-32.

${ }^{17}$ Zhou, K., Doyle, J.C., and Glover, K., Robust and Optimal Control, Prentice-Hall, Englewood Cliffs, NJ, 1996.

${ }^{18}$ Gahinet, P., Nemirovskii, A., Laub, A.J., and Chilali, M., LMI Control Toolbox Mathworks, Natick, MA, 1995.

${ }^{19}$ Nguyen, L.T., Ogburn, M.E., Gillert, W.P., Kibler, K.S., Brown, P.W., and Deal, P.L., "Simulator Study of Stall/PostStall Characteristics of A Fighter Airplane with Relaxed Longitudinal Static Stability," NASA Technical Paper 1538, 1979.

${ }^{20}$ Stevens, B.L., and Lewis, F.L., Aircraft Control and Simulation, John Wiley \& Sons, Inc., 1992.

${ }^{21}$ Buffington, J.M., Sparks, A.G., and Banda, S.S., "Robust Longitudinal Axis Flight Control for An Aircraft with Thrust Vectoring," Automatica, Vol. 30, No. 10, 1994, pp. 1527-1540.

${ }^{22}$ Shin, J.-Y., Balas, G.J., and Kaya, A.M., "Blending Approach of Linear Parameter Varying Control Synthesis for F-16 Aircraft," AIA A Paper 01-4040, Aug. 2001.

${ }^{23}$ Buffington, J.M., and Enns, D.F., "Flight Control for Mixed-Amplitude Commands," International Journal of Control, Vol. 68, No. 6, 1997, pp. 1209-1229.

${ }^{24}$ Hammett K.D., Reigelsperger W.C., and Banda S.S., "High Angle of Attack Short Period Flight Control Design with Thrust Vectoring," Proceedings of 1995 American Control Conference, Seattle, Washington, June 1995, pp. 170-174.

${ }^{25}$ Reigelsperger, W.C., Hammett K.D., and Banda S.S., "Robust Control Law Design for Lateral-Directional Modes of An F-16/MATV Using $\mu$-Synthesis and Dynamic Inversion," International Journal of Robust and Nonlinear Control, Vol. 7, No. 8, 1997, pp. 777-795.

${ }^{26}$ Boyd, S.P., El Ghaoui, L., Feron, E., and Balakrishnan, V., Linear Matrix Inequalities in Systems and Control Theory, SIAM, Philadelphia, PA, 1994.

${ }^{27}$ Gahinet, P., "Explicit Controller Formulas for LMI-Based $\mathcal{H}_{\infty}$ Synthesis," Automatica, Vol. 32, No. 7, 1996, pp. 10071014 . 